\title{
Strategy for the updating of the school education content in the chemical information noosphere civilization
}

\author{
Svetlana A. Volkova, ${ }^{1, *}$ \\ ISED RAE, Center of Science Education, 105062, Moscow, Russia
}

\begin{abstract}
The article deals with the didactic aspects of the new strategy for updating the content of school education in the chemical information noosphere civilization. The author proposes a mechanism for updating the content of education and work in the information and educational space for the integration of modern science in the content of chemical education.
\end{abstract}

The modern world has changed significantly. Now there is the trend to call it cyberspace, virtual reality, "fifth dimension", the telecommunications space. This qualitatively new world has changed, and the man himself has changed as well. Space changed its functioning, perception, consciousness, and thinking. According to Academician V.I. Vernadsky there is a new shell around the Earth - "noosphere" (sphere of reason), in which the information management and organization are the main engines of progress. The mass computerization, the introduction and development of information technologies, the emergence of digital educational resources (DER) has led to a dramatic development of not only research, but also education. Under the DER we understand specially formed units of various information resources for use in the learning (educational) process shown in digital (electronic) form and functioning on the basis of information and communication technologies (ICT). These include virtual labs, interactive whiteboards, electronic resources, trainers, e-learning modules and other tools $[1,2]$. Information has become a global, in principle, inexhaustible resource of humankind, which requires intensive development of new information noosphere civilization.

The changes occurring in the world require a completely new philosophical understanding of the past, present and future for the entire human civilization. Its development strategy should be looked at in terms of connections to informatics achievements, cybernetics, genetics, synergy, microelectronics, environmental, as well as rapidly developing nanoscale science and supramolecular chemistry. This allows us to apply the new multi-level methodology of cognition and learning process - a methodology for information civilization [3]. As the leading methodological approaches we apply a systematic approach, understanding it as a systemic impact of modern science to philosophy, and civilization is directly related to the ideas of scientific rationality.

In recent years, the phenomenon of rationality is not only up to date, but also became the "nail", around which philosophical thought is currently concentrated. What caused such a deep interest in the problem of scientific rationality?

Under the scientific rationality in the most general sense we understand the system of closed and selfsufficient rules, norms standards and algorithms, universally valid and accepted as part of the society in order to achieve meaningful social purposes. Selfsufficiency and isolation means that the participants of the system accept and understand this rationale, believe that this ensures the achievement of certain conscious or unconscious purpose, without the involvement of other regulations, standards and norms. This does not exclude that in the process of development associated with a change in goals, it made a certain correction for sound. At the same time, it is stored for a steady, stable kernel, which is not subject to little or no modification and is subject to change. It provides its operation and development, while maintaining self-identity. With the change in the goals and values set of rules, standards and norms that make up this rationality, it begins to be evaluated by representatives of this or any other community of people as the irrational. The standards and norms, including the ideals of a special variety of standards, with a particular "reading" can be translated into the language of the rules. This makes them dominant in all rationality.

Rationalism has arisen primarily in the sphere of science and scientific activity, as the most efficient system is science, scientific knowledge. In the Age of Enlightenment, there existed three types of scientific rationality, which corresponded to the three major stages of the evolution of science, replaced by each other in the framework of the development of industrial civilization:

1. The era of classical science. Within its framework, the classical type of rationality focuses only on the subject and makes the brackets everything that relates to the subject of this science and the means of its activity.

2. The era of non-classical science. Here rationality is seen in the context of relativity, the idea of the object to the media activities and operations, since the latter are 
the condition for obtaining true knowledge about the object.

3. The era of post-nonclassical science. Postnonclassical rationality takes into account the correlation of knowledge about the object not only with means but also with the values and structures of the target activity.

At present, the phenomenon of technological civilization and rationality enters a period of a particular type of progress when humanistic orientation is directed towards original scientific research in determining strategy. This provision suggests considering the rationality of the educational process in the unity with the humanistic orientations of education as a priority in its development [4]. Research conducted by scientists of the Institute of Developmental Physiology, Russian Academy of Education, shows that the training is carried out at the expense of health. Teaching the student is very difficult, as the state of his emotional status - fear. By this cause, there appears permanent time trouble in the conditions of the temporary stressors that violate the physical and mental health [5]. Therefore, we need new approaches, forms, methods, didactic means, the contents of which should be multi-system [6].

The challenges of modern society dictate, firstly, the "avalanche" of increasing volume of information to be the assimilation and comprehension of students, and secondly, the emergence of new requirements of the educational standard (GEF), associated with a change in the understanding and "indicator" estimation of quality of training school graduate, updating the problem of the integration of modern science in chemical content of school education. The ability to adequately respond to these challenges is largely determined by the President and the Government: to improve the efficiency of scientific research, the development of highly scientific manufacturing technologies as a means of economic development. The technological breakthrough of the second half of the $20^{\text {th }}$ century, followed by a "renaissance" of industrialization require training, development of science, development of new technologies "that will change the world, the nature of the economy, way of life of millions, if not billions of people." Under these conditions, the new strategy is becoming a systematic update of educational content based on the achievements of modern science.

Pedagogical science and practice should answer the following questions: What kind of content should be covered by school textbook, to improve education, increase scientific literacy and culture of growing people, not to the era of "impoverishment of the soul while enriching the information?" How to reflect in the objective content the relationship of these forms of life such as science, art, from which man draws knowledge? How should we structure this content to solve the problem of preservation of logical thinking in an age of virtual dominance of information, in particular, information on the work of knowledge, its transformation into knowledge? What principles should the creation of electronic textbooks and manuals be based on? What didactic functions appear in a new generation of textbooks? How to integrate the new content of education in the information-subject environment, including complex DER e-learning materials, which, in turn, influence the content of updated training? We should bear in mind that the content of education should reflect all the latest achievements of chemical science, chemistry associated with the successes, problems and troubles of modern society, the latest advances in science technology.

We offer a mechanism for updating the content of education and activities, comprising the following steps:

- The first stage involves the global structuring of educational material in order to isolate the optimal structure of invariant knowledge and its visual presentation (models, model and natural experiment, the media and others.) for the further use as benchmarks of the expected qualitative learning outcomes;

- The second stage - the local structuring systems of chemical concepts and generalized skills within each large block of content in order to determine in it structural and functional, genetically related, as well as the methodological and practical significance of the new components of content, expressed in terms of thematic, conceptual and instrumental complexes of organization teachers and students;

- The third stage consists in the consolidation of didactic units of knowledge and modes of action due to coagulation, compression of information in compact symbolic and graphic forms of expression and operation; the use of different kinds of materialization and formalization of the training based on the principle of minimizing knowledge. This principle is reflected in the fact that at a minimum the typical objects considered in different aspects, different phenomena are studied and form chemical concepts and generalized skills. In addition, there should be options for content blocks with coiled information, its further development and possible use of interactivity;

- On the fourth stage we assume generalization, theoretical generalization and systematization, intra - and interdisciplinary integration, the categorical synthesis and transfer of system knowledge and skills generalized algorithmization means computerization, technologizing, with the active use of cybernetic and mathematical methods to describe the structure of knowledge and the quality of their assimilation. In this regard, we see a clear priority of multimedia in combination with full-scale model and a chemical experiment.

Many years of our experience in school practice show that the implementation of this mechanism in the information-educational environment is possible in two directions: the inclusion of knowledge of current research and scientific achievements in the content, both general and further education. Additional education develops intensively in the direction of the development of numerous elective courses on relevant topics. We consider the possibility of integration of knowledge about the achievements of modern science in chemical content of school education.

Didactic units of knowledge are concepts. Chemical concepts as the core content of education have a greater share of abstraction as a concentrate of essential, generalized knowledge expressed in the form of an 
iconic model, which comes to mind, summing up the results of the knowledge of essential features, properties, relationships and regularities and chemical reactions [7]. Updating the content of teaching chemistry, we distinguish in it the nodal components, the language of artificial intelligence; carry out "meaning granulation." As such "semantic pellets" are the interconnected system of chemical concepts. On the other hand, the emergence of new content blocks associated with the development of a new style of training, involving a comprehensive analysis of the substantive aspect of the educational process and the optimal combination of educational technology training, the choice of its methods, techniques, tools, and organizational forms.

Chemical substance is the main concept of chemistry. Substances surround us are everywhere: in the air, soil, household appliances, food, plants, and finally, in ourselves. People synthesized the largest number of substances, which previously did not exist in nature, i.e. advanced materials, pharmaceuticals, and catalysts. To date, there are about 20 million of organic and inorganic substances, and each of them has its inner structure. Organic and inorganic synthesis reached a high degree of development that allows synthesizing any compound with a predetermined structure. In this regard, at the forefront of modern chemistry, we see an applied aspect in which the focus is on the communication structure of matter with its properties, and the main task is to find and synthesis of useful substances and materials with desired properties. Currently, a young multidisciplinary branch of modern science develops rapidly in the chemistry of the future - supramolecular chemistry. What is a "supramolecular" chemistry?

The founder of Supramolecular Chemistry - Nobel Prize winner, the eminent French scientist J.-M. Lehn. In 1978, he wrote, «... chemistry of molecular assemblies and of the intermolecular bond»; in 1988 «Supramolecular chemistry is the chemistry of the intermolecular bond»; 2002 - «Supramolecular chemistry aim at development highly complex chemistry systems from components interacting by non-covalent intermolecular forces». Thus, supramolecular chemistry is the chemistry of the intermolecular bonds. Just as there is a range of molecular chemistry based on covalent bonds, there is also the area of supramolecular chemistry, chemistry of molecular ensembles and intermolecular bonds [8].

Students know that chemistry studies the chemical elements (atoms), they form molecules (crystals), their transformation. Isolated molecule is the exception rather than the rule, an abstraction rather than a reality.

Supramolecular chemistry studies:

- environment role in chemical reactions;

- molecules are composed of groups: synthesis of ensembles of molecules, intermolecular interactions, the properties of the molecules in the collective properties of the team as a whole, the analysis and the collective description of the structure, the creation of devices based on molecular groups (molecular electronics), imitation and study of biological processes, the impact of conditions in the ensembles molecules.
Supramolecular chemistry began with the study of the selective binding of alkali metal cations natural and synthetic macrocyclic and macropolycyclic ligands, crown ethers and cryptands. Research field expanded, leading to the realization of molecular recognition as a new field of chemical research, which is putting the spotlight of intermolecular interactions and processes in general, spreading to the whole spectrum of related areas, connected with supramolecular chemistry [7].

Problem field of supramolecular chemistry as a science includes the following research areas:

- (1): 1. Identify the existence of intermolecular bonds and the determination of their energy characteristics (using spectroscopic methods - IR, Raman, inelastic neutron scattering). 2. Identify the existence of cross-linking and finding their geometrical characteristics (using diffraction methods). 3. Statistical study of intermolecular interactions based on analysis of the data collected for a large array of compounds.

- (2): 1 . The use of the intermolecular interactions in the synthesis of chemical compounds (new classes of complex organic molecules, coordination compounds, composites, biochemical synthesis). 2. Use of intermolecular interactions for the design and synthesis of new crystalline structures (Engineering crystals), films, liquid crystals, membranes, micelles, as well as their modification. 3. Use of intermolecular interactions for the design and synthesis of new materials with desired properties or modifying existing materials.

- (3): 1. Investigation of the role of intermolecular interactions and supramolecular organization in the formation of the physical properties (anisotropy response to external stimuli, such as temperature, pressure, magnetic properties, color, etc.). 2. Modeling of intermolecular interactions for the enthalpy and entropy calculations to predict the polymorphism: existence, preparation conditions and properties of the maximum possible number of polymorphs. 3. Experimental and theoretical study of the role of intermolecular interactions in phase transitions and chemical reactions involving supramolecular assemblies.

Research methods of supramolecules are the following: nuclear magnetic resonance, mass spectroscopy, thermogravimetric analysis, differential scanning calorimetry, scanning microscopy. For example, the study of supramolecular structures of magnetic resonance techniques, including electron spin resonance, allows defining the geometry of the complex.

Applications supramolecular compounds are varied. For example, the porphyrins are a model of assimilation by the body of light, photosynthesis. They also represent a remarkable reversible oxygen binder, almost indifferent to carbon monoxide. New technology storage at the molecular level using rotaxane molecules would bring the storage density to 100 gigabits per square inch.

Academician A.I. Konovalov states that the universe is evolving towards an increasing complexity, from less complex to more complex systems. The ability to form more complex systems lies in the nature of the less complex. Thus, the formation of atoms is the property of elementary particles; the formation of molecules is the property of atoms. If there is a molecule that will make 
supramolecular systems, and if there is a supramolecular system, then there will be biological systems, therefore, there will be life. Supramolecular Systems are the highest point of prebiotic evolution. They are a bridge from nonliving matter to live: there are biological systems based on supramolecular systems.

Supramolecular chemistry affects issues related to borders and the essence of life. One of them concerns the origin of the biological world; the second issue concerns the possibility of the existence of life beyond the Earth and the Solar system. The meaning of the third question is trying to understand why life forms are shaped in the way that we know; whether it is possible then to create an artificial life. Thus, supramolecular chemistry is an interdisciplinary field of science, including chemical, physical and biological aspects of the consideration of more complex, than molecules of chemical-related systems into a single unit by means of intermolecular non-covalent interactions.

Another interdisciplinary field of science is developing nanoscience and nanotechnology on its basis. Changes in the modern world are largely due to fundamental and applied research structures with qualitatively new properties that arise due to changes in the size of the structural elements to the nanometer scale, with the technology for their production, follow-up work with them, i.e., with nanotechnology. Accordingly, the new trends in science should be reflected in the content of school education, as in the formation of modern holistic view of the world is obvious the special role of nanotechnology in the scientific and technological progress at the present stage and the scientific concepts of nanoobjects and interrelated phenomena. The problem nanostate substance is one of the most urgent problems of modern science, which lies at the intersection of materials science, physics, chemistry and computer technology. The popularity of nanotechnology and connected with it rapid development of all areas of science, studying nano-objects, influenced school education in the last few years.

In recent years, students have to cope with an enormous flow of information associated with the creation of materials, devices, household items, titles or descriptions for which there is the prefix "nano". We are talking about nanotubes, nanocrystals, nanorobots, nanothermometer nanovesah, nanomotors, nanofibers, nanowires, and others. Such terms as "Nanoparticles", "Nanochemistry", "Nanotechnologies" can be found in the headlines and magazine articles, radio and television almost every day. Among these disciplines, Nanochemistry relates to the section chemistry, exploring the properties, structure and characteristics of chemical transformations of nanoparticles.

Nanotechnology is the key concept of the beginning of the $21^{\text {st }}$ century, a symbol of the new, third scientific and technological revolution. Its development holds great promise in the development of new materials, the improvement of communication, the development of biotechnology, microelectronics, energy, health and weapons. Among the most likely scientific breakthroughs experts forsee a significant increase in computer performance, the recovery of human organs with the use of the newly reconstituted tissue, obtaining new materials, created directly from the set of atoms and molecules, as well as new discoveries in chemistry and physics. Nanotechnology is a combination of production methods with a given atomic structure by manipulating atoms and molecules.

We recommend including in the content of both basic and further education the information about the latest achievements of Supramolecular Chemistry and nanoscale science. The content of the lessons of chemistry should include videos, quality and settlement problems. For additional education, we have developed the concept and the program of e-learning course on relevant topics.

The concept is the idea of integrating knowledge about nanotechnologies and nanomaterials in natural sciences additional education. Electronic training course are created in SCORM-format modules, which will cover an extensive area of expertise, including interdisciplinary field school program. Nanotechnologies have a significant impact on the development of the educational system, by initiating changes in traditional approaches to the organization of educational process. To ensure the quality of the development of such programs and their subsequent effective implementation it is necessary to integrate the knowledge about nanotechnologies and nanomaterials in science education using distance learning technologies. This distance education has become an integral component of modern national education systems, determining their competitiveness on the international market of educational services, and the possibility of their implementation of the regulations attached.

The learning process must be built in accordance with the "natural way of knowledge. The use of "real objects or, at least, of their images" is important. It is necessary to focus on the development of content related to personal observations of students, with the results of their perception, organizing and understanding, as well as modeling, allowing to build models and to "see" objects of the nanoworld. For this purpose, organization of virtual tours in the nanoworld, the use of different multimedia components of the educational process becomes the means of educational process. The theoretical content can be included in the format of video lectures with extensive use of both traditional forms and methods of learning, and new, such as a virtual experiment, computer simulation, etc. Initially, during the study of each module, it is advisable to include short introductory lectures (rollers) of leading experts in each field of science.

The implementation of the proposed concept in the form of e-learning course in SCORM-format modules in additional education system in Russia is the challenge of time, in response to which the natural sciences can bring schooling to the level that meets the high requirements of the twenty-first century. Because of e-learning, the students will obtain knowledge and the possibility of its use in the life and practice [9].

\section{References}

1. S.A. Volkova, and S.O. Pustovit, SC, 4 (2013)

2. S.A. Volkova, and S.N. Gusev, SC, 6 (2010) 
3. R.F. Abdeev, Philosophy of information civilization (Moscow, 1994) [In Rus]

4. S.A. Volkova, Domestic education. Integration of moral education in various disciplines, 4 (2010)

5. M. M. Bezrukikh, Report on the meeting of the Academic Council from 29.02.2016 (ISED, Moscow, 2016) [In Rus]

6. T.S. Nazarova, Instrumental didactics: Advanced tools, environment, and technology training (Moscow, 2012) [In Rus]

7. N.E. Kuznetsova, Formation of concepts in modern training systems chemistry: Textbook (Moscow, 1985) [In Rus]

8. J.-M. Lehn, Supramolecular Chemistry: Concepts and perspectives (Moscow, 1998)

9. S.A. Volkova. Integration of knowledge about nanotechnology in the content of science education (to be published) 